\title{
Apprendre la mer au Gabon et défier les approches disciplinaires
}

Learning the ocean in Gabon and challenging disciplinary approaches

\section{Catherine Sabinot}

\section{OpenEdition}

\section{Journals}

\section{Édition électronique}

URL : http://journals.openedition.org/ethnoecologie/6212

DOI : $10.4000 /$ ethnoecologie. 6212

ISSN : 2267-2419

Éditeur

Laboratoire Eco-anthropologie et Ethnobiologie

\section{Référence électronique}

Catherine Sabinot, «Apprendre la mer au Gabon et défier les approches disciplinaires », Revue d'ethnoécologie [En ligne], 18| 2020, mis en ligne le 28 décembre 2020, consulté le 29 janvier 2021. URL : http://journals.openedition.org/ethnoecologie/6212 ; DOI : https://doi.org/10.4000/ ethnoecologie.6212

Ce document a été généré automatiquement le 29 janvier 2021.

\section{(c) (i) (9)}

Revue d'ethnoécologie est mis à disposition selon les termes de la licence Creative Commons Attribution - Pas d'Utilisation Commerciale - Pas de Modification 4.0 International. 


\title{
Apprendre la mer au Gabon et défier les approches disciplinaires
}

Learning the ocean in Gabon and challenging disciplinary approaches

\author{
Catherine Sabinot
}

1 Il est un lieu commun dont on peut faire le constat dans de nombreux écrits et discours tenus sur les différents continents: "tout se perd...». Les valeurs, les langues, les "traditions », les savoir-faire, sont affectés. Peut-on toutefois parler uniquement de perte des savoirs? Serait-ce si simple ? Nos recherches nous conduisent à aller au-delà de cette impression de perte, et à appréhender ce qui guide et nourrit la dynamique des savoirs et savoir-faire, véritable poumon des transformations des sociétés qui s'adaptent aux changements environnementaux, socio-économiques, culturels et politiques auxquels elles font face.

Lorsqu'en 2003, Serge Bahuchet, alors porteur d'une action concertée incitative (ACI) visant à comprendre comment les populations acquièrent, adoptent et partagent les savoirs, m'a donné l'opportunité de nourrir cette réflexion au travers d'une thèse de doctorat en ethnoécologie sous sa direction, j'ai eu la bonne idée de la saisir! Sur les littoraux du Gabon, je me suis attachée à décrire des dynamiques culturelles variées et à décrypter les mécanismes de transformation et de transmission des savoirs des sociétés de pêcheurs qui y résident. Cet article entend rendre compte des avancées menées durant ces quatre riches années de thèse au sein de l'UMR Éco-anthropologie et Ethnobiolgie et de mettre en avant l'intérêt d'articuler des approches anthropologiques et ethnoécologiques aux outils des autres disciplines pour appréhender la dynamique des savoirs avec un regard nouveau.

Serge avait une grande connaissance des sociétés forestières d'Afrique centrale et avait encore peu travaillé sur la pêche ou le milieu marin (Bahuchet 1985, 1993). Néanmoins, il m'a encouragée à poursuivre mes recherches sur les littoraux que j'étudiais depuis le début de mon cursus universitaire et à travailler avec les pêcheurs côtiers du Gabon sur lesquels très peu d'écrits existaient. Les littoraux, espaces d'interfaces tant écologiques qu'humaines, sont en effet des "laboratoires de choix» pour appréhender la dynamique des savoirs. De tout temps peuplés ou traversés par des hommes de cultures 
et de langues différentes, ils sont des lieux où savoirs, savoir-faire et représentations symboliques sont en constante tension. Certains changements dans ces espaces plus sujets à la rencontre de l'Autre parfois très lointain, sont eux-mêmes précurseurs de changements à l'intérieur des terres. L'étude de la dynamique des savoirs dans les communautés natives et migrantes de pêcheurs du Gabon, s'est accompagnée du montage avec Serge, Sylvie Lebomin, Jean-Emile Mbot puis Jean-Marie Betsch d'un partenariat solide et durable entre le Muséum national d'Histoire naturelle de Paris et l'Université Omar Bongo de Libreville. L'ethnoécologie, et plus généralement les ethnosciences, y ont eu un rôle essentiel, tant dans les enseignements que dans les thèses de master et de doctorat que le partenariat a fait émerger. Ce contexte a donc été aussi l'opportunité d'accompagner des étudiants gabonais et français dans leurs recherches au Gabon et de partager les avancées de nos propres recherches.

En décrivant les relations que les hommes construisent avec les autres êtres vivants, humains et non-humains, mes travaux doctoraux, profondément ancrés dans l'ethnoécologie ${ }^{1}$, ont questionné les dynamiques culturelles se jouant sur le littoral gabonais, dans le quotidien des interactions avec l'environnement, dans le quotidien de la formation, de l'acquisition et de la transmission des savoirs et des savoir-faire. Ils ont aussi posé, par la suite, les premières pierres d'un chemin de recherche qui m'a conduit sur les littoraux et îles de l'océan Atlantique et du bassin Indo-Pacifique à étudier les savoirs écologiques locaux et à rendre compte de leur intérêt pour appréhender les transformations socio-environnementales (Sabinot 2007, Doyon \& Sabinot 2015, Sabinot \& Bernard 2016, Sabinot \& Lescureux 2019, Sabinot \& Herrenschmidt 2019).

5 Cet article décrira les choix méthodologiques faits durant la recherche doctorale que Serge Bahuchet a encouragée et présentera les étapes suivies ainsi que leurs principaux résultats: l'approche ethnographique globale des communautés installées sur les $800 \mathrm{~km}$ de la côte gabonaise, l'étude plus locale des relations que trois communautés entretiennent avec leur environnement dans la région de Mayumba à la frontière congolaise, puis l'étude fine systématique d'objets culturels phares qui a mis au jour des dynamiques culturelles, propres à chaque communauté comme spécifiques aux interactions inter-communautaires, et a mis en exergue ce qui structurait ces dynamiques.

\section{Une approche ethnographique dessinant l'organisation du littoral à l'échelle nationale}

6 Durant les deux premiers mois du terrain de thèse, une démarche à la fois prospective et descriptive des différents rapports à l'océan existants, et des échanges et des liens existant entre des groupes a priori isolés les uns des autres, a été adoptée. Des entretiens dirigés et semi-dirigés doublés d'une observation participante des modes de vie, des compositions des villages, des connaissances du milieu, des pratiques liées à l'eau, et des représentations du monde aquatique, ont permis d'appréhender les communautés ainsi que les réseaux d'échanges qu'elles animent, et de rendre compte d'une organisation à l'échelle nationale, des communautés gabonaises d'une part, des groupes de migrants d'autre part, ainsi que des interrelations qui s'y tissent.

7 Les littoraux africains sont caractérisés par une forte proportion de migrants, souvent pêcheurs, saisonniers ou non (Rouch 1950, Jorion 1979, Durand et al. 1991, Bouju 2000, 
Dia 2001, Marfaing 2005). Les Gabonais, bien que disposant de 800 kilomètres de côte, ont toujours très peu exploité la ressource maritime. Mais comme en atteste la figure 1 , de nombreux pêcheurs étrangers se sont installés sur les côtes depuis la fin du XIX siècle.

Figure 1 : Principales migrations de pêche de la fin du XIX ${ }^{e}$ siècle aux années 1980

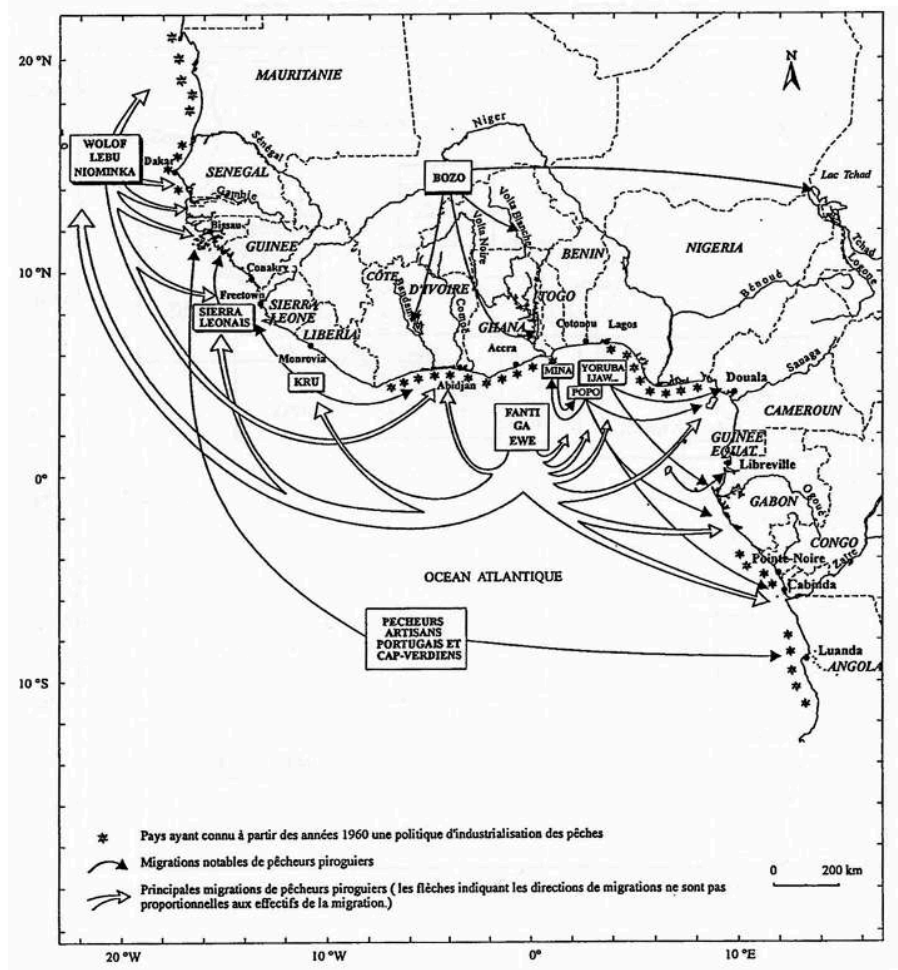

D’après Chauveau (Chauveau \& Weber 1991).

8 Aujourd'hui, les habitants ouest-africains des pays du golfe de Guinée (Ghana, Togo, Bénin, Nigéria) sont les plus présents au Gabon et sont réputés très bons pêcheurs comme en attestent les témoignages recueillis en 2004 et 2005.

«Je suis venu au Gabon pour la pêche! Quand on est Popo, quand il y a du poisson dans un pays, on y va!» (Homme béninois de 50 ans, Port-Gentil, le 21/12/2004)

«Les Nigérians, ils pêchent seulement. Tout ce qui ne vient pas de l'eau, ils achètent. » (Homme fang gabonais de 42 ans, Cocobeach, le 05/01/2005)

9 Ainsi, océan et lagunes gabonaises voient se côtoyer différentes communautés (Figure 2) : les Africains côtiers de l'ouest pêchent en mer tandis que les Gabonais et quelques Sénégalais pêchent en lagune. 
Figure 2 : Répartition des étrangers sur le littoral gabonais ${ }^{2}$

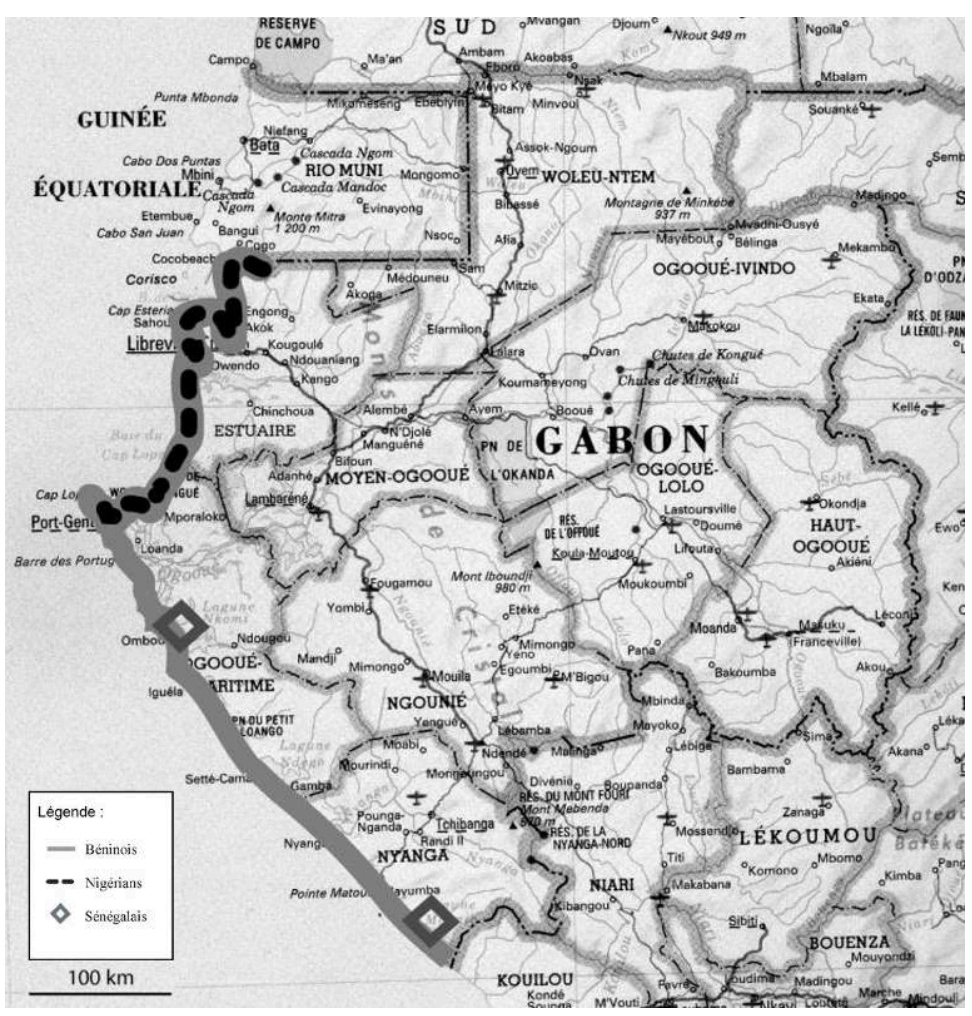

10 À l'échelle nationale, entre les communautés de pêcheurs étrangers en mer, des échanges d'outils, de travailleurs et/ou de techniques sont courants et indispensables. Les échanges de savoirs et de savoir-faire, notamment naturalistes, se font directement par des déplacements d'hommes, mais aussi par voie écrite, ou plus récemment à travers l'usage de téléphones cellulaires largement répandus (Sabinot 2008).

\section{Une étude des pêcheurs du bassin de la Banio, espace pluriculturel tourné vers la mer et la lagune}

11 Fort de notre compréhension de ce qui se joue à l'échelle nationale entre les communautés de pêche, une étude localisée dans un espace pluriculturel restreint a été réalisée, non à Libreville ou Port-Gentil, villes où beaucoup d'éléments s'enchevêtrent, où les influences internes comme externes sont difficiles à identifier et à dissocier, non sur un espace où une grande quantité de communautés cohabitent, mais au sud du Gabon dans un milieu où trois communautés principales sont amenées à construire ensemble leur espace de vie.

Dans la Province de la Nyanga, le bassin de la Haute et Basse-Banio accueille environ 3500 personnes. Par la présence de seulement trois communautés principales (de nationalités béninoise, sénégalaise et gabonaise), il facilitait l'observation et l'analyse des dynamiques culturelles (Figure 3). 
Figure 3 : Répartition des communautés sur les berges de la lagune Banio (Sabinot 2007)

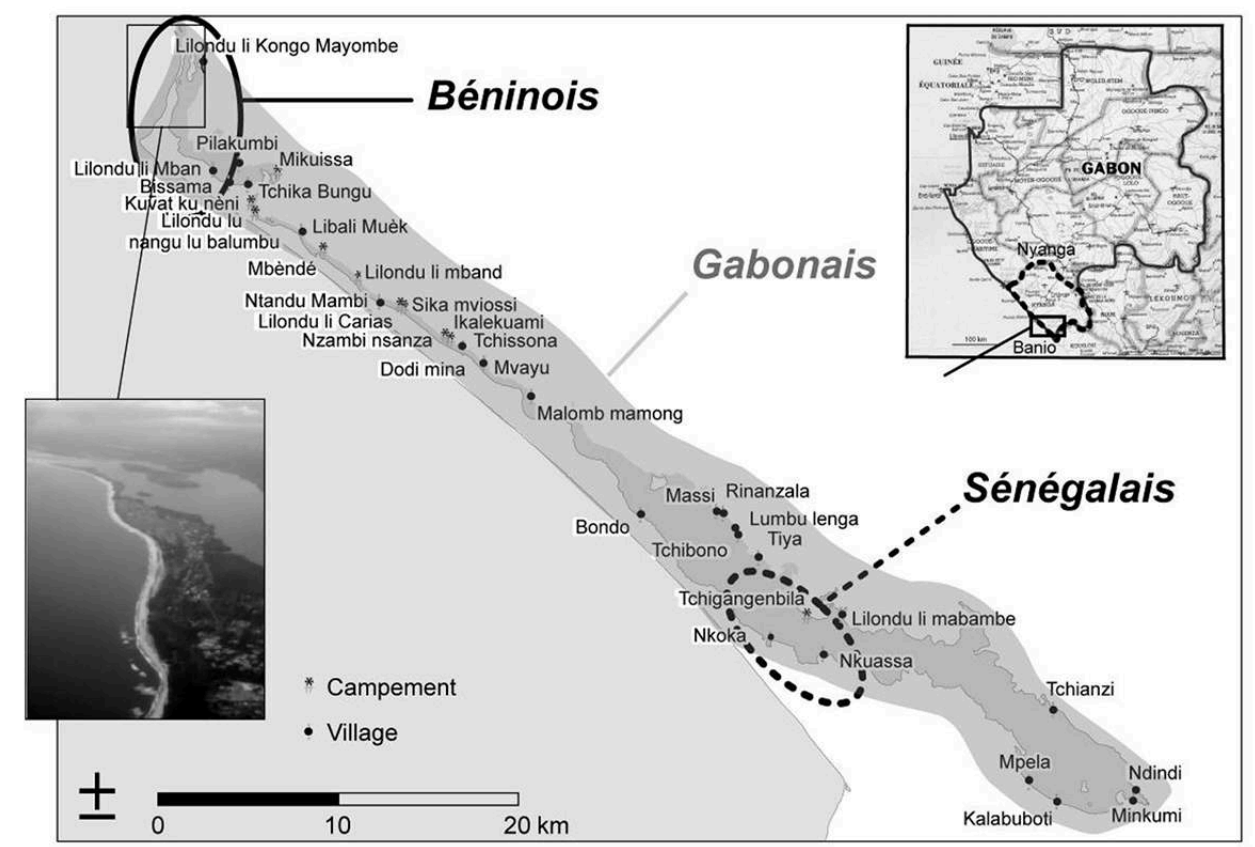

Les Gabonais, Vili et Lumbu principalement, sont présents sur l'ensemble de la lagune ${ }^{3}$. Plus de 2000 individus sont installés dans la commune de Mayumba à l'embouchure, tandis que les autres se regroupent en petits villages ou campements le long des berges de la lagune. Les Béninois, Phla principalement ${ }^{4}$, se sont rassemblés à l'extrémité nord de Mayumba, à l'embouchure. Ils y ont créé leur quartier : «Tchiole Ndembé ». Certains foyers gabonais y résident, ainsi que quelques femmes gabonaises mariées avec des hommes béninois. Quant aux Sénégalais, de langue fulfulde, ils ont créé leur quartier dans un village de la lagune, Nkoka.

Cette répartition des locaux, des Béninois et des Sénégalais n'est pas anodine puisqu'elle se répète au sein des autres lagunes littorales du Gabon. Les Béninois à l'embouchure pêchent en mer, les Gabonais des campements pêchent en rivière et lagune, les Sénégalais pêchent en lagune. Ainsi, qu'elles soient le fait de migrants ou le fait d'autochtones suivant les mouvements des ressources aquatiques, les migrations de pêche construisent l'occupation du littoral, selon les années et selon les saisons. 
Figure 4 : Départ des pêcheurs béninois de Mayumba depuis la plage de L'Office

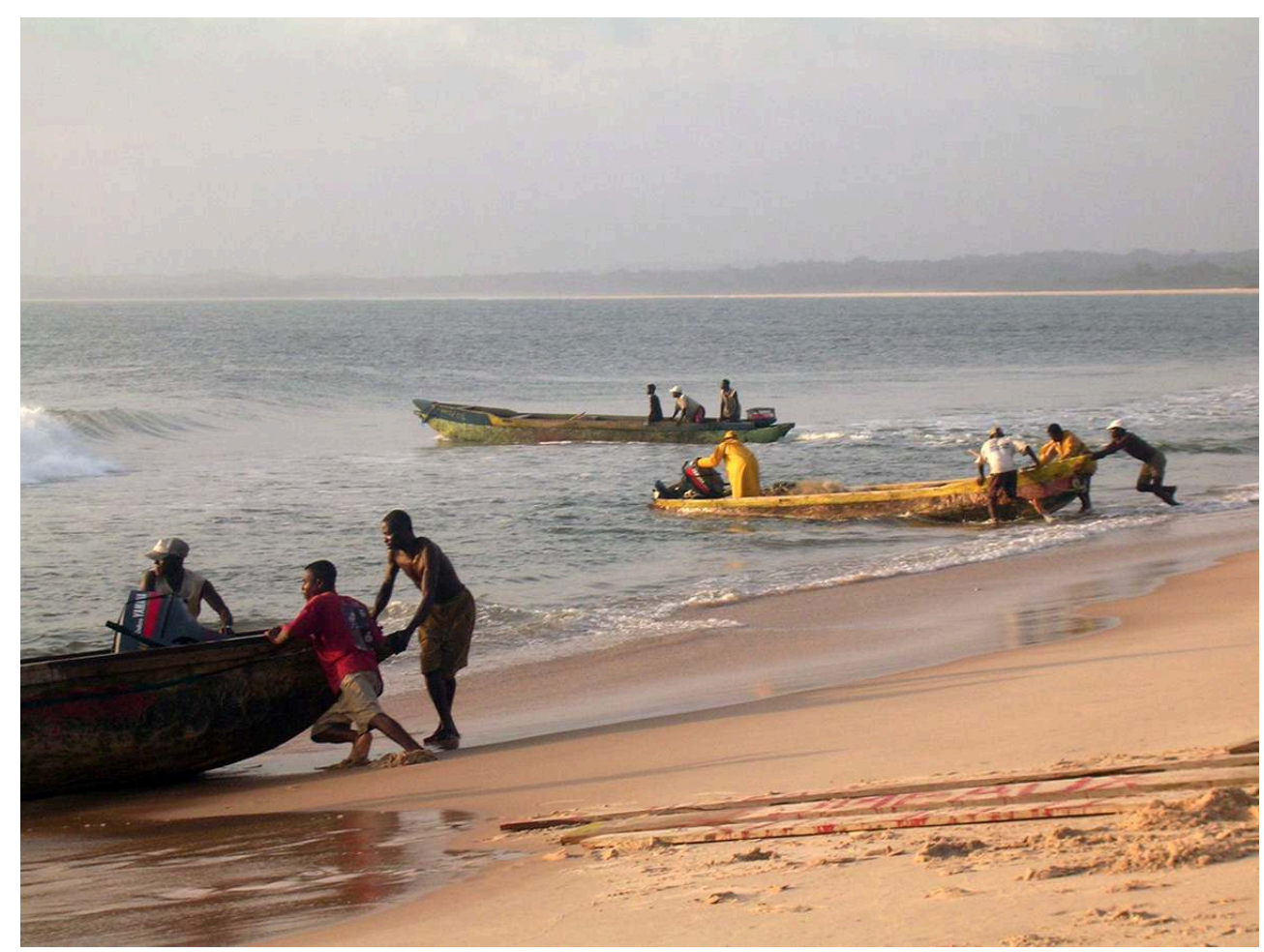

(C) C. Sabinot 2004

15 Autour de la lagune Banio, chaque communauté a mis en place un «système littoral » qui lui est propre et se caractérise par un système d'acquisition, de transformation et de consommation des éléments qui l'entourent, un système de production, un système de relations à la famille et à la communauté, un système de relations à l'invisible. Puis face à l'Autre, aux Autres, face à leurs savoirs, leurs savoir-faire, leurs manières de percevoir et de concevoir, les individus et les communautés sont progressivement invités à transformer certains de leurs objets culturels, à choisir de ne pas en modifier d'autres, pour des raisons techniques et identitaires. 
Figure 5 : Pêche à la senne dans la lagune Banio près de l'embouchure

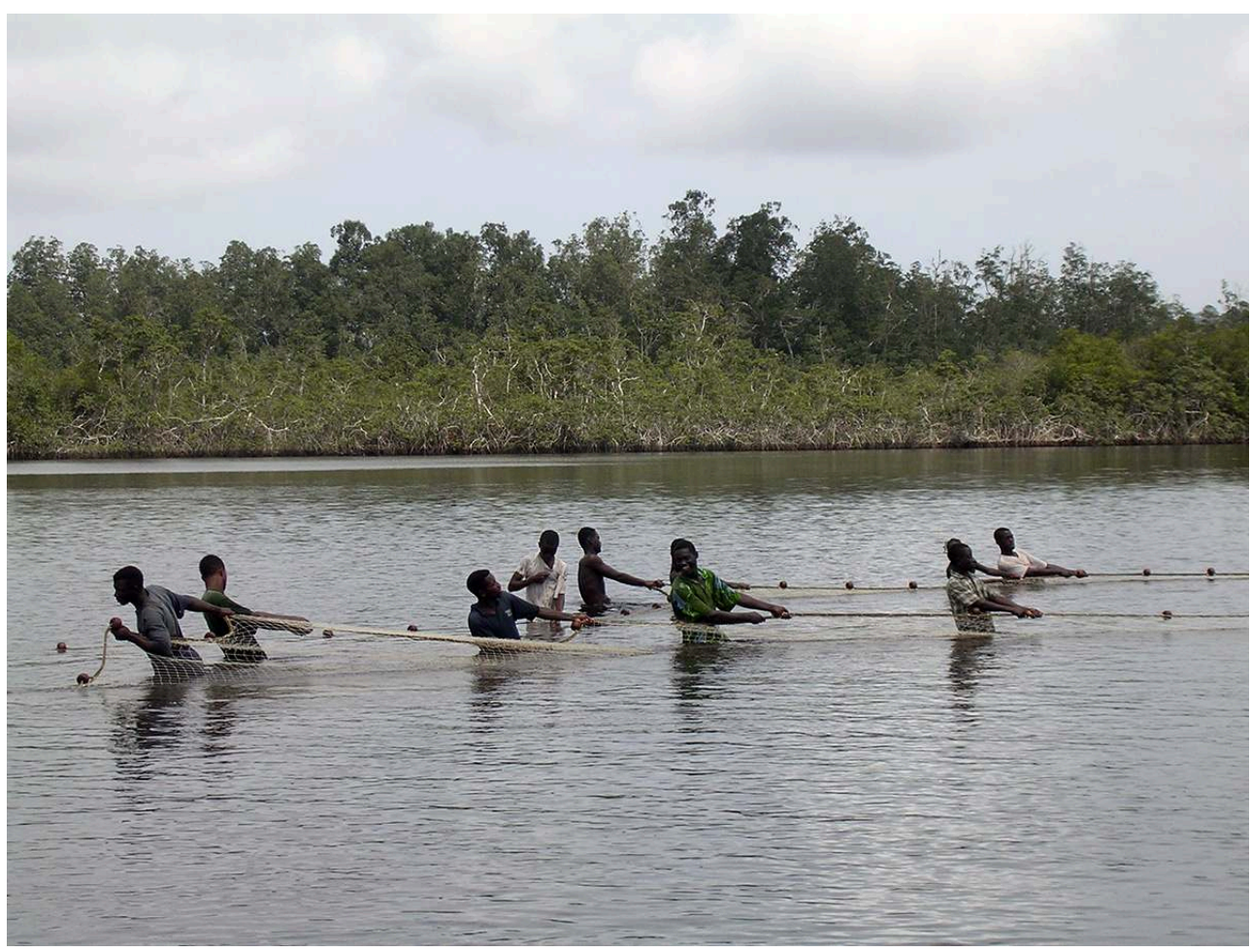

(C) C. Sabinot 2004

16 Sur ce territoire, des échanges commerciaux et linguistiques, des mariages mixtes, des échanges de savoirs, de savoir-faire, de savoir-être se font. Les communautés autochtones et migrantes se sont construites réciproquement et forment un espace de vie né des interrelations entre les humains et entre les humains et les non-humains. Pour analyser la construction de l'espace géographique, un relevé exhaustif des toponymes de la lagune Banio a été réalisé. ${ }^{5}$ Cette analyse toponymique a permis de révéler certaines relations que les hommes entretenaient, d'une part avec leur environnement, et d'autre part avec les groupes culturels habitant un même espace. Trois cent un toponymes ainsi que la motivation de leur appellation ont été collectés : un site peut devoir son nom aux caractéristiques écologiques du milieu (comme Malondu ma mvubu, les débarcadères des hippopotames), à ses caractéristiques historiques, ou encore à ses caractéristiques mythiques (Mbulu lutchieni, la pointe qui ne fane pas, où réside un génie du même nom). Toutes ces motivations classées en plusieurs niveaux ont permis de faire des cartes thématiques et des diagrammes révélateurs de nombreuses interactions entre les hommes et leurs environnements (Sabinot 2008). Les figures 6 et 7 représentent ainsi les origines historiques des toponymes, et permettent d'apprécier comment divers groupes migrants se sont succédé et ont contribué à nommer les sites de la lagune. 
Figure 6 : Carte des toponymes de la lagune Banio qui trouvent leur origine dans l'histoire des lieux, liée aux Vili, Lumbu, Pygmées, Congolais, aux colons ou aux missionnaires.

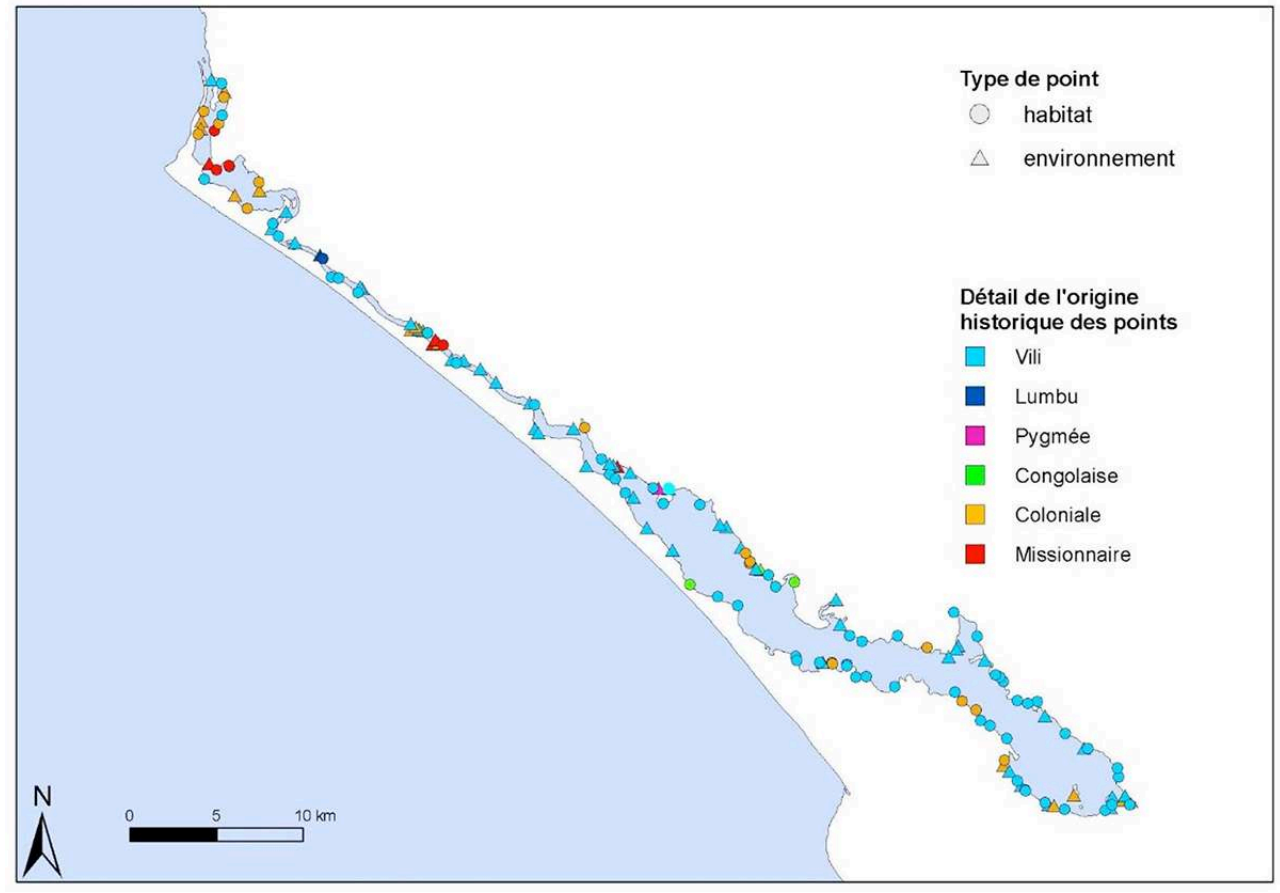

Figure 7 : Origine historique du linéaire côtier de la lagune Banio

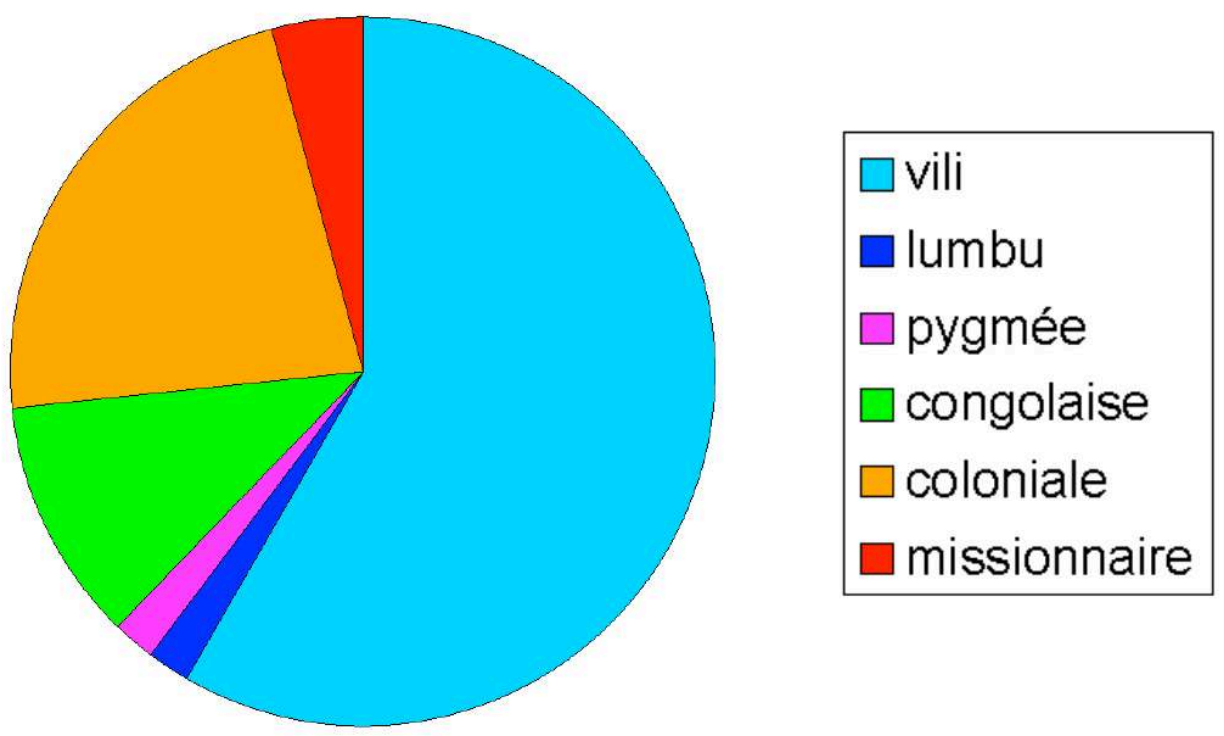

17 Considérant que le paysage rend compte du processus interactif entre l'homme et son environnement (Ingold 1993: 152), et que le vocabulaire en témoigne, l'analyse toponymique a ainsi permis d'approcher diachroniquement et synchroniquement les relations que les hommes ont entretenues et entretiennent avec cet espace. 


\section{Des objets culturels phares pour saisir les dynamiques culturelles et appréhender ce qui les structure}

18

Afin de saisir la complexité de la dynamique des savoirs et d'en identifier des éléments structurants, au-delà de l'ethnographie du bassin de la Banio et de ses habitants, un travail ciblant dix objets culturels phares - car éclairant la compréhension des processus de transmission des savoirs communs aux groupes de pêcheurs - a été conduit.

Ces objets ne sont pas de simples objets techniques mais des supports de savoirs: l'étude de chacun a donc été l'étude de savoirs relatifs aux systèmes de production, d'utilisation, de commercialisation, et de pensée, liés à des modalités d'apprentissage variables.

Les objets culturels phares composent une série représentative des types de savoirs présents sur le territoire étudié. Ils ont été au nombre de dix : hameçon, pirogue, filets (filet maillant et épervier) - moyens de conserver le poisson (viviers, fumoirs, claies de séchage), nage, génies des eaux, coquillages. Pour satisfaire à leur vocation de représentativité, les objets-phares devaient répondre à divers critères, relatifs notamment au contexte social de la pratique et de l'apprentissage définis par six éléments: le genre - chaque objet se distingue dans sa pratique par son caractère dominant masculin (pirogue, filet, nage), féminin (coquillages) ou mixte (vivier, génie, hameçon); les générations - certains objets sont plus spécifiques d'une tranche d'âge (génie, vivier), d'autres caractérisent quelques générations (fumoir, salaison, nage), d'autres sont communs à toutes (hameçon, coquillage, pirogue); les communautés de pratique $^{6}$ - certains objets s'expriment plus particulièrement dans un contexte familial, amical, entre travailleurs, ou entre étrangers, d'autres s'utilisent au sein de différentes communautés de pratiques; le statut identitaire ou non de l'objet - il peut s'agir d'un objet partagé peu distinct d'une communauté à l'autre ou d'un objet marqueur d'identité (statut identitaire fort des fumoirs ou des génies qui offrent à chacun le moyen de se reconnaître d'une communauté) ; l'importance apparente de l'agency ${ }^{7}$, degré de liberté dont dispose chaque acteur au sein de la communauté et répercussions que ses initiatives peuvent avoir sur les autres habitants; le type de mémoire (individuelle ou collective) mise en jeu par l'objet - certains objets sont l'attribut d'individus spécialistes, de groupes sociaux ou font partie de la mémoire commune. Les objets ont aussi été choisis pour représenter différents contextes techniques, définis de manière contrastée par leur complexité - petite ou grande (l'hameçon est un objet simple, l'épervier un objet plus complexe, nécessitant l'assemblage de nombreux matériaux différents), l'importance de l'environnement technique (l'utilisation ou la fabrication de la pirogue demandent un environnement technique conséquent contrairement à la nage ou aux génies), le type de technique mise en ceuvre - technique du corps ou technique instrumentale, et la fonctionnalité de l'objet - unique ou multiple (les claies de séchage ont une seule fonction alors que la pirogue en a plusieurs). Le contexte écologique a quant à lui été défini par le lieu où "s'exprime » l'objet (mer, lagune, depuis la terre ou sur l'eau), le lieu de son apprentissage et le caractère saisonnier ou non de son utilisation. Enfin, les objets devaient représenter une diversité de contextes cosmologiques marqués entre autres par des relations à l'invisible gérées par quatre types d'interdits - liés aux rapports sexuels, à la période des menstrues, à la possession de pièces de monnaie, ou encore au contact avec d'autres espèces animales ${ }^{8}$. 
21 Les objets culturels phares furent aussi choisis pour représenter différentes modalités de la transmission des savoirs et différents types d'évolutions. Une attention a été portée au mode de transmission (verticale ou horizontale) - construire une pirogue se transmet de père en fils, apprendre à nager se fait entre pairs ; au fonctionnement de la transmission des savoirs: enseignement direct par la parole et/ou les gestes, apprentissage par l'observation non "guidée ", apprentissage par l'expérience, écoute de mythes, de récits réels; au sens de la transmission (unique ou réciproque); aux échanges constatés entre les communautés - certains objets sont l'objet d'échanges entre nationalités (pirogue, vivier, fumoir, salaison), d'autres ne le sont pas a priori (coquillages, hameçon, génie, nage, filet); à l'innovation, la réhabilitation et/ou la rétention - innovation forte pour les viviers / rétention forte pour les fumoirs; au changement technique; à l'innovation cognitive et la créativité.

22 Au regard de ces critères, chaque objet a été analysé au travers des discours des praticiens et des non-praticiens de chaque communauté. Considérer un ensemble d'objets très différents et envisager leur comparaison, a exigé une contextualisation méthodique de ce qui les caractérise, et donc la réalisation en amont de questionnaires systématiques. Une attention spécifique a été portée sur la manière dont chacun voit son apprentissage, son enseignement et ceux des autres, tant dans la "conception » que dans "l'utilisation" de l'objet culturel. Deux types de questions essentielles portant sur la dynamique culturelle étaient toujours posées : «- Qui vous a appris et à qui apprenez-vous la production et l'utilisation de cet objet? » et «- Quelles évolutions avez-vous constatées? Quelles en étaient les causes ?».

Interroger la relation que chaque informateur entretient avec les objets a permis de déterminer les contextes de la formation, de l'acquisition, de la transformation des savoirs et des savoir-faire, puis de lister les éléments changeants pouvant avoir une influence sur les objets culturels. Ont ainsi été mises au jour un certain nombre d'évolutions, permettant de décrire des dynamiques culturelles types telles que celles décrites ci-dessous.

\section{Transformation du filet et de la pratique avec l'arrivée d'un matériau}

En traitant de l'objet phare filet, les conséquences de l'arrivée d'un nouveau matériau ont été décrites.

Vers 1930, les fils de coton importés par les Portugais ont été rapidement adoptés par les Gabonais de la Banio car cette matière remplaçait avantageusement les fibres végétales employées auparavant pour réaliser une nappe de filet. L'outil s'est transformé par la matière qui le composait mais aussi par les dimensions plus importantes que cette matière permettait.

Cette modification a entraîné des évolutions des techniques de pêche, car la manipulation d'un filet de plusieurs centaines de mètres est différente de celle d'un filet de quelque vingt mètres; elle a aussi conduit à un changement des lieux et des temps consacrés à la pratique, et a modifié le nombre de pêcheurs participant à l'activité. Le nouveau matériau a donc permis l'évolution de l'outil, qui à son tour a entraîné l'évolution de la technique, puis de la pratique.

Le changement de matériau entraîne parfois une modification de l'objet dans sa conception; le changement de l'outil peut quant à lui entraîner le bouleversement de tout un système. Si ce bouleversement est accepté car il correspond techniquement et 
«identitairement » à la communauté, l'objet sera adopté. Dans le cas contraire, si les blocages culturels sont forts, une résistance intense se mettra en place et le nouveau matériau ou outil ne sera pas adopté par l'ensemble de la communauté.

Figure 8 : Débarquement des sardinelles à Mayumba

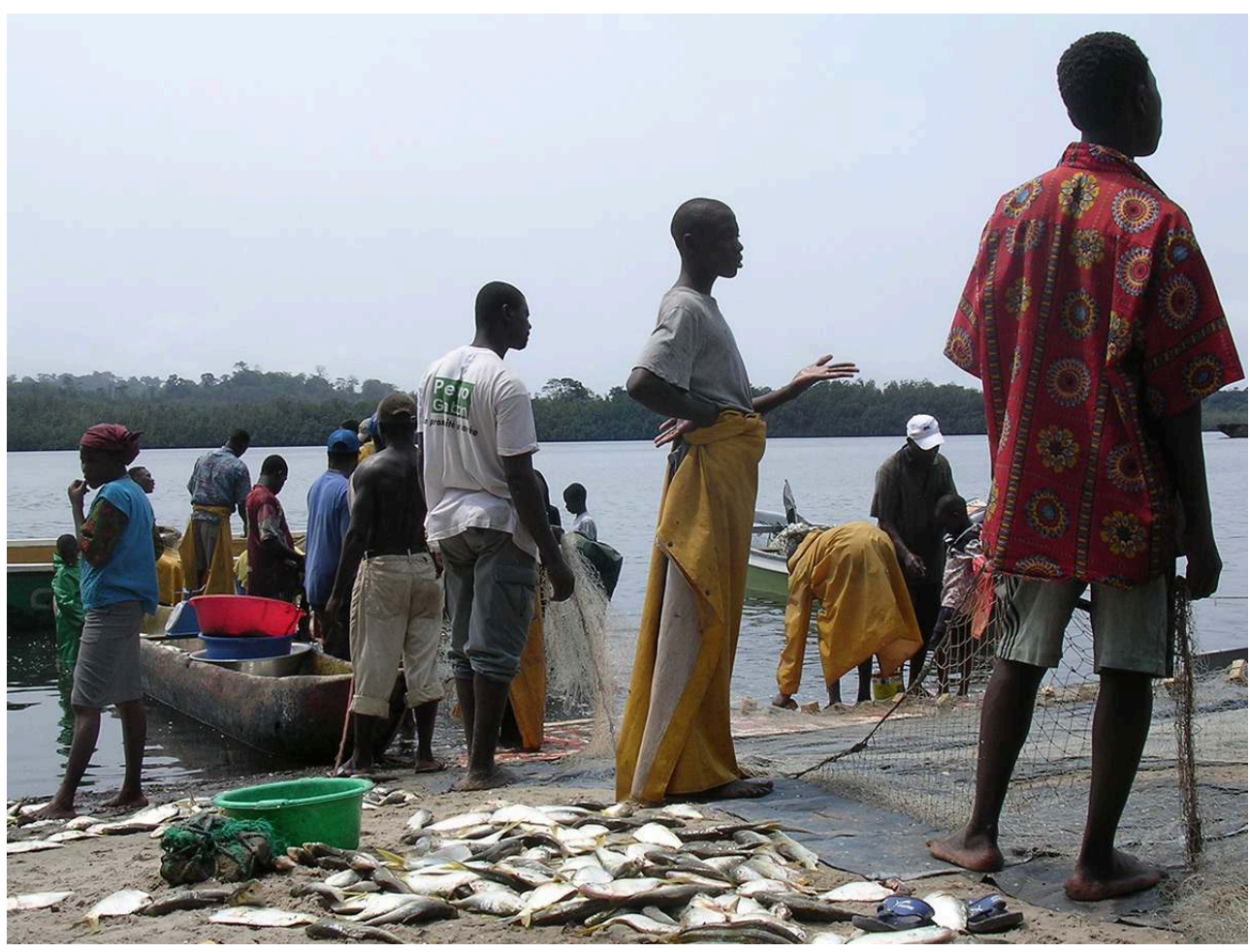

(c) C. Sabinot 2006

\section{Changement de genre dans les pratiques de collecte des coquillages}

Certains changements n'ont pas été déclenchés par un seul phénomène bien identifié par mes informateurs, mais par la conjugaison de plusieurs facteurs. Ainsi en est-il de la collecte des huîtres auparavant exclusivement réservée aux femmes.

Le contexte cosmologique a subi des transformations qui ont eu des conséquences sur les activités aquatiques. Ainsi, quel que soit le sexe des individus, la collecte des huitres, initialement pratiquée après avoir obtenu l'autorisation du " maître de l'embouchure ", se réalise dorénavant en passant outre cette procédure. En outre, certaines femmes enfreignent désormais les interdits liés à leur état menstruel, pour rester « compétitives».

De plus, le contexte économique s'est modifié, offrant aux huîtres une valeur pécuniaire importante et un espace de vente national. Par ailleurs, les mutations du contexte écologique, d'une part liées aux transformations du savoir-être des individus dont nous venons de faire état, et d'autre part conséquentes aux mouvements cycliques «naturels » des bancs de sable, ont abouti à un déplacement des huîtres vers les zones plus profondes de la lagune. D'après certains informateurs masculins, cette nouvelle conformation, s'ajoutant à la rentabilité financière, justifie la présence des hommes 
dans l'activité, puisqu'ils se considèrent physiquement supérieurs aux femmes. Aujourd'hui, ils se consacrent intensément à la " plonge des huîtres " pendant la saison sèche.

Figure 9 : Préparation des huîtres collectées en plongée par les femmes de l'office à Mayumba

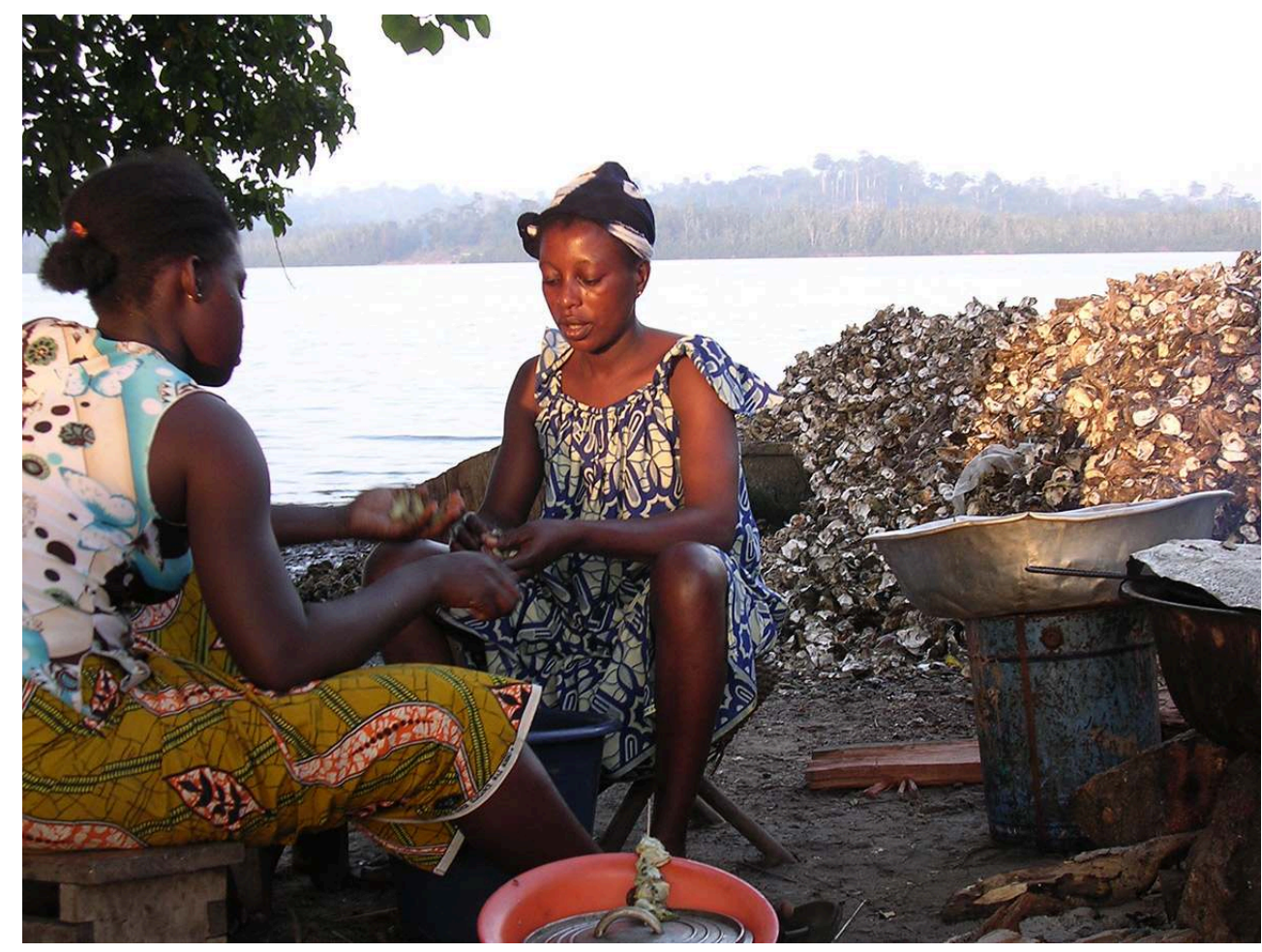

(c) C. Sabinot 2006 
Figure 10 : Femmes et hommes "plongeant les huîtres" dans la lagune à Mayumba

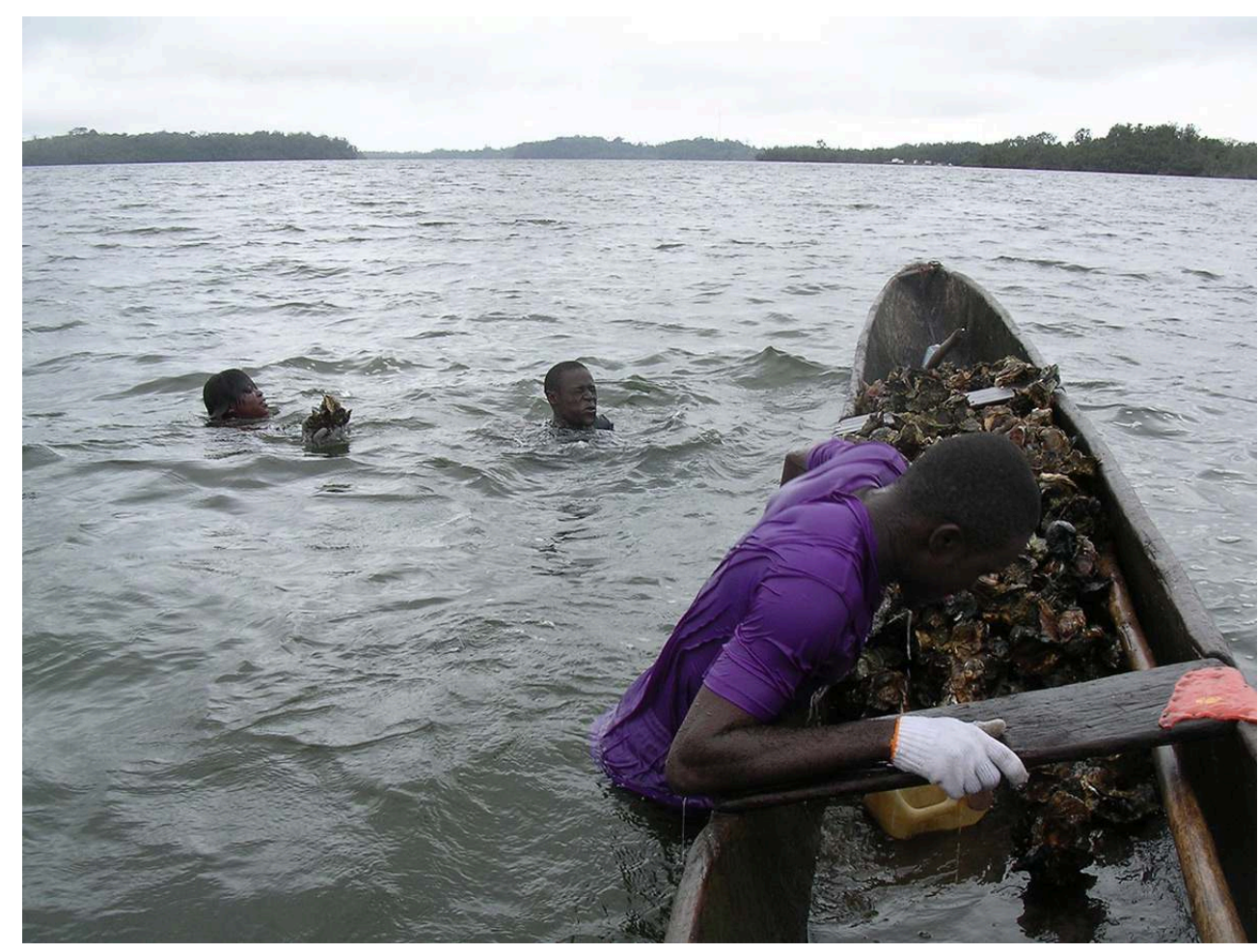

(C) C. Sabinot 2006

31 En raison des modifications des contextes économique, cosmologique et écologique, la composition sociale des communautés de pratique a donc changé, tout comme les caractéristiques temporelles et spatiales de l'activité. En conséquence, les modalités de transmission de ce savoir-faire ont aussi été transformées. L'objet culturel qui se transmettait entre femmes se transmet désormais d'une femme vers un homme, ou d'un homme vers une femme, et même entre hommes. L'objet qui se transmettait des femmes plus âgées vers les plus jeunes, soit de manière verticale, se transmet aussi aujourd'hui de manière horizontale, entre alter ego. Enfin, alors que l'apprentissage se réalisait dans le cercle familial, il se réalise aujourd'hui également dans le cercle amical.

32 Le genre, les générations et les réseaux de relation sont donc nettement bouleversés au cours de l'évolution de cet objet culturel, sans qu'il soit évident de distinguer quelles sont les influences respectives que peut avoir chaque modification de contextes sur ces évolutions. Les dynamiques de changement sont créées par la mise en résonance de plusieurs modifications de contextes.

\section{Transformation des modalités de construction des pirogues}

33 Une autre conjonction de modifications de contexte a créé autour de la pirogue une autre dynamique : un refus d'emprunt associé à un transfert de savoir-faire.

34 Les pirogues gabonaises étant peu résistantes à la houle marine, et les arbres de la forêt nécessaires à la fabrication d'une pirogue appartenant aux autochtones, les Béninois ont dû dépasser les contraintes territoriales et sociales pour disposer d'embarcations adaptées à leurs besoins. Ainsi, depuis que les pirogues originaires de leur région ne sont plus en état d'aller en mer, quelques hommes béninois compétents ont entrepris 
d'apprendre aux Vili comment monter un bordage sur une pirogue, comment rehausser de planches les bords de l'embarcation originairement monoxyle. Désormais, les Vili creusent donc les pirogues comme leur ont appris leurs aînés, mais ils les rehaussent de planches comme leur ont enseigné les pêcheurs migrants. On assiste ici à un enseignement direct de l'étranger vers l'autochtone afin de disposer du matériel auquel il est habitué, celui que son activité nécessite.

Le besoin technique apparaissant au cœur d'une conjonction de contextes spécifiques s'est révélé créateur de liens en suscitant la naissance de diverses interrelations entre Gabonais et Béninois: échange de connaissance sur le bois d'œuvre, transfert de compétence, emploi, commerce, et nouveaux rapports d'influence.

De plus, une transmission de savoirs spécifiquement verticale, de père en fils ou d'oncle en neveu, s'est partiellement transformée en transmission horizontale: un homme béninois enseigne à son homologue gabonais. La modification de l'objet culturel s'accompagne donc de nouveau d'une mutation des modalités de sa transmission.

\section{Définir, comprendre et repérer des types de dynamiques culturelles}

Après avoir finement observé, questionné et décrit chaque objet culturel phare, ont été identifiées, schématisées et analysées toutes les dynamiques repérées. Puis, souhaitant aller plus loin dans l'analyse, souhaitant accéder à une meilleure compréhension de la manière dont les populations acquièrent, emploient et partagent leurs savoirs et savoir-faire, nous avons osé conjuguer d'autres échelles d'observation et d'analyse, tout en éprouvant un nouvel outil offrant un autre regard sur nos résultats, avec la connivence de Jean-François Ponge, Jean-Marie Betsch et Bruno Toupance. Deux questions principales se sont posées: comment les modifications de contextes environnementaux, écologiques, techniques ou humains entrent-elles en synergie pour entraîner du changement ou du non-changement des objets culturels, comme des modalités de leur transmission? Des tendances peuvent-elles être dégagées?

$\mathrm{Au}$ cours des entretiens menés au Gabon, sept types de changements et dix-sept raisons les expliquant ont été avancés par nos interlocuteurs. En plus de décrire les raisons explicatives des évolutions, la plupart des individus interviewés précisaient les personnes qu'ils reconnaissaient comme étant à l'origine de ces changements (Béninois, Sénégalais, missionnaires, colons). Les changements identifiés, affectant les savoirs et les savoir-faire ainsi que les modalités de leur transmission, sont les suivants : changement dans le genre des praticiens (ex : un objet culturel exclusivement féminin devient mixte); dans la relation à l'invisible (ex : un objet culturel géré par le monde invisible subit une modification); changement technique, mécanique (ex : un objet culturel est transformé dans sa conception ou son utilisation technique); changement de l'âge de praticiens (ex: une activité réservée aux adultes devient pratiquée par les enfants); de l'usage de la ressource (ex : un objet culturel existant pour l'autosubsistance devient objet de commerce); dans la relation aux autres humains (ex: un objet culturel est investi par d'autres individus qui sont de fait considérés autrement) ; dans l'intensité de la pratique (ex : un objet culturel est moins sollicité, voire disparaît). 

dissimulés, incontournables ou insignifiants au chercheur. Par souci d'objectivité, seuls les 17 facteurs cités par les habitants ont été retenus: le besoin d'argent, l'augmentation de la ressource, la transgression des tabous, les capacités personnelles, l'époque, l'économie du temps et de l'effort, la qualité de l'objet, l'arrivée de l'électricité, la motorisation, la transformation de l'environnement physique, les esprits, la loi, un nouveau matériau ou matériel, la migration, la compétition entre pairs, ou encore les medias (télévision, radio, Internet, téléphone).

Après avoir listé ces différents éléments, il s'est agi d'organiser toutes les données afin de comprendre comment celles-ci étaient liées, comment elles entraient en synergie pour créer des dynamiques culturelles. En anthropologie, pour identifier cette synergie, nous nous basons sur nos observations, nos enquêtes, l'analyse des histoires de vie. Cette approche, pour accéder à un regard global, nécessite de passer de nombreuses années parmi les différentes communautés. Au travail ethnographique, indispensable, a été adossée l'utilisation d'un outil nouveau pour l'ethnoécologue, permettant d'offrir une représentation plus lisible, et de mettre en évidence des hypothèses non apparentes de prime abord: les analyses factorielles des correspondances $(\mathrm{AFC})$. Cet outil a déjà fait ses preuves et est très utilisé dans les sciences biologiques, mais il n'avait pas encore été sollicité pour répondre au type de questionnement que nous nous posions9.

Disposant d'une masse de données conséquentes et collectées systématiquement sur les savoirs, les savoir-faire et leurs évolutions, les AFC ont assisté notre exploration à ambition comparative, car elles permettent de traiter une grande quantité de données qualitatives, de favoriser une vision d'ensemble des données en les rendant lisibles sous forme graphique, et de suggérer des hypothèses pouvant expliquer ce qui structure les données.

Ainsi, le traitement par AFC des données ethnoécologiques organisées sous forme de matrice, a permis de regrouper des éléments et d'en isoler d'autres pour leur singularité. Puis, il a suggéré des structures d'organisation des savoirs sur le territoire étudié. La figure 11 représente, par exemple, un des schémas d'organisation extrait d'une analyse factorielle. La disposition des nuages de points sur le graphique AFC a permis de suggérer des regroupements, nous amenant à assembler des types de changements d'une part (à gauche) et des causes de changements d'autre part (à droite). 
Figure 11 : Représentation schématique de la mise en parallèle des types de changements et des causes les ayant engendrés

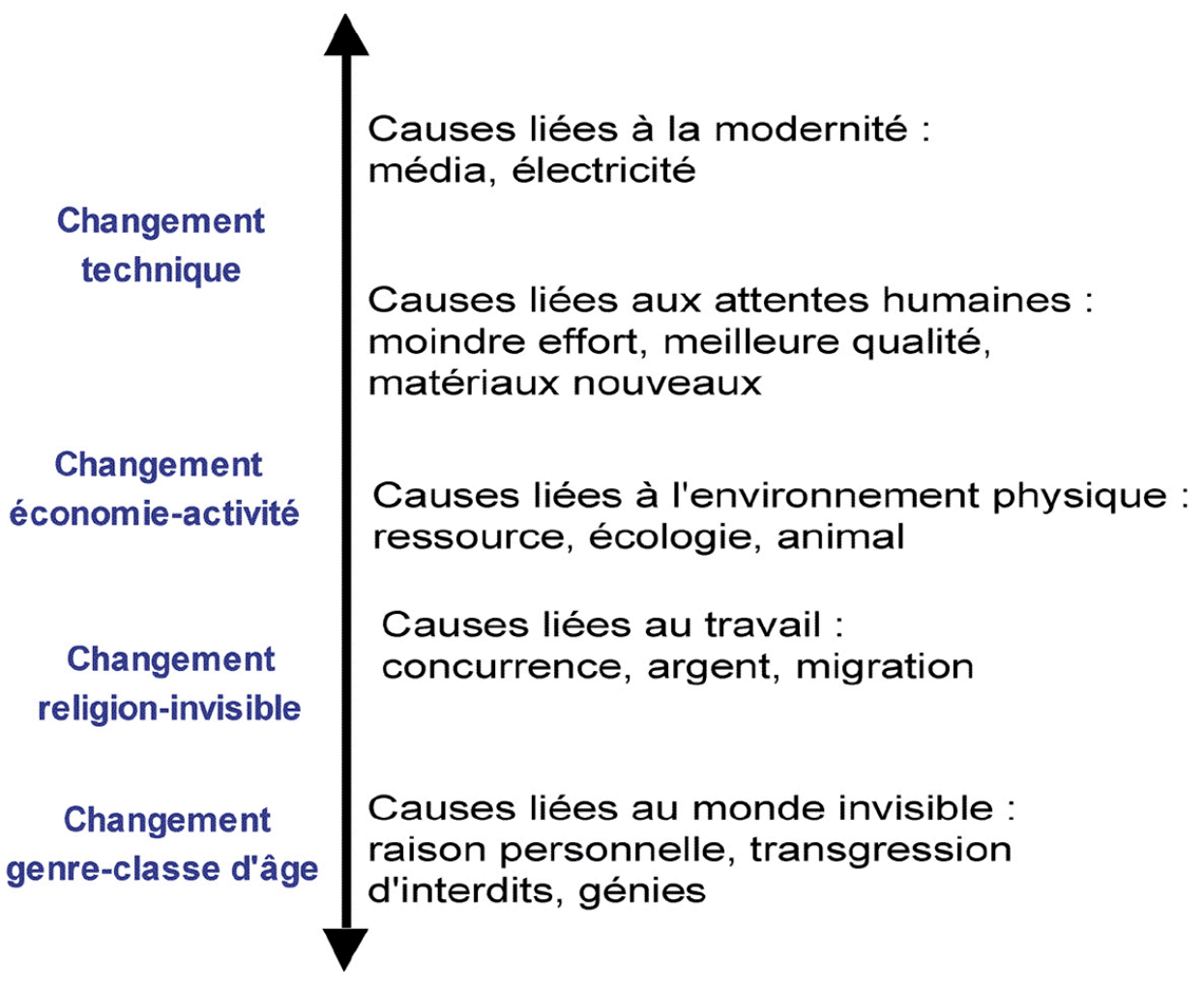

Les résultats de cette AFC interrogent les liens de cause à effet, et permettent de classer les objets selon la manière dont ceux-ci évoluent. Ces conclusions, possibles à partir d'un travail basé sur dix objets, le seraient également si nous travaillions sur une centaine d'objets culturels, et c'est là tout le potentiel des analyses factorielles. Le chercheur ne pose pas les premières hypothèses mais se les fait suggérer par l'analyse, ce qui permet de ne pas partir sur des idées reçues.

$\mathrm{Au}$ final, la mise en résonance des contextes de la pratique et de l'apprentissage finement définis et des éléments descriptifs de leur évolution, ont révélé des tendances évolutives propres à chaque type de savoir, qui peuvent se révéler très intéressantes pour déterminer les évolutions possibles d'un objet culturel non encore documenté. Les représentations graphiques obtenues (référencées dans Sabinot 2008) ont notamment permis de poser deux hypothèses. Un objet transmis de manière verticale, qui est important dans le quotidien des adultes, souvent une technique instrumentale, semble plus à même de subir une modification technique. En revanche, un objet transmis de manière horizontale, qui est important dans le quotidien des enfants, souvent une technique du corps, semble plus à même d'engendrer une modification de l'organisation de la société. D'autres analyses factorielles semblent confirmer également, comme quelques auteurs l'avaient déjà spécifié (Acerbi \& Parisi 2006; Hewlett \& Cavalli-Sforza 1986), que des savoirs qui se transmettent verticalement sont plus rétifs au changement que ceux qui se transmettent horizontalement.

Au cours de cette recherche, nous avons montré que les modifications de certains éléments de contexte peuvent non seulement avoir des répercussions sur les savoirs mais aussi sur les processus de leur transmission. Au-delà de refléter une grande diversité de dynamiques culturelles, nous avons donc progressé dans la compréhension 
globale de la dynamique des savoirs, sur les types d'impacts que peuvent avoir les modifications de l'environnement humain, technique ou écologique. Au niveau humain et technique, la disparition, l'émigration ou l'immigration d'individus détenteurs de savoirs ou de savoir-faire, les modifications de la composition sociale des groupes migrants, la genèse de nouveaux comportements et de nouveaux savoir-être, venus de l'intérieur ou initiés par les individus et les médias "extérieurs ", ont souvent généré des transferts, voire des substitutions de territoire et de compétences. Quelques emprunts ont été constatés mais aussi des refus d'emprunt, dans le cas des pratiques de boucanage par exemple. Les modifications de l'environnement écologique ont également provoqué des emprunts, souvent rapides, auxquels se sont combinées des imitations. Ainsi en est-il des "viviers des Sénégalais » façonnés en réponse à un comportement inattendu d'une espèce pêchée en s'inspirant des viviers fabriqués par les autochtones, ou des éperviers des enfants gabonais en réponse à une modification du milieu aquatique. Ces jeunes ont inventé un épervier de petite dimension, allégé en plomb, afin de pêcher des poissons vivant désormais dans un volume d'eau très réduit juste au-dessus d'un fond vaseux (Sabinot 2007).

\section{Conclusion}

46 Au Gabon, savoirs, savoir-faire et savoir-être ont été ajustés par les pêcheurs locaux et migrants en vue de vivre mieux dans leurs environnements. Nos travaux anthropologiques décrivent finement ces changements et montrent que les pertes strictes de savoirs sont finalement rares et peuvent plus justement s'appréhender comme des transformations de savoirs dépendantes de modifications techniques, écologiques, sociales et cosmologiques. Des bouleversements, des variations ou des changements quasi imperceptibles, au sein des communautés et entre elles, ont été observés. La variété des évolutions présentées, des jeux de facteurs en partie décelés, montre l'infinie complexité de la dynamique des savoirs, reflet de l'extraordinaire complexité de l'Homme, individuellement, collectivement, et encore plus lorsqu'il entre en interaction avec d'autres sociétés que la sienne.

Aussi, les étapes méthodologiques choisies pour étudier les transformations des pratiques comme des modalités de leur transmission ont marqué le jury de la thèse de doctorat soutenue en 2008 pour leur originalité et le défi transdisciplinaire qu'elles engageaient. Avec l'appui de Serge Bahuchet et de l'équipe qu'il dirigeait, un outil, les AFC, a été éprouvé et adapté aux types de données que nous pouvons collecter en ethnoécologie et en anthropologie. Une analyse d'objets culturels phares, supports de savoirs, permet d'avancer des hypothèses sur l'organisation des savoirs et des savoirfaire, et offre de nouvelles pistes d'interprétations et de nouvelles questions de recherche. Approcher la dynamique des savoirs par un regard ethnoécologique progressif, doublé d'une démarche analytique la plus indépendante possible de nos a priori culturels nécessite de provoquer l'interface entre les disciplines, les outils et méthodes propres à chacune, car c'est à la frontière de plusieurs disciplines que peut se construire une telle réflexion, de conjuguer des échelles d'observation et d'analyse, de favoriser les démarches comparatives. Pour ce faire, nos outils d'analyses et nos méthodes de transcriptions gagnent à être enrichis et bousculés, défi qui est de plus en plus élevé (entre autres Soengas 2010; Reyes-García 2007, 2014). À l'heure où les réflexions sur l'évolution de l'homme et de son environnement sont sans cesse 
discutées, il est essentiel de poursuivre notre compréhension des processus qui gouvernent la transmission des savoirs, car ils jouent un rôle primordial dans les évolutions de nos relations avec la biodiversité, particulièrement à long terme.

\section{BIBLIOGRAPHIE}

Acerbi A. \& Parisi D. 2006 - Cultural transmission between and within generations. Journal of artificial societies and social simulations 9 (1). [En ligne] : http://jasss.soc.surrey.ac.uk/9/1/9.html

Bahuchet S. 1985 - Les Pygmées Aka et la forêt centrafricaine. Ethnologie écologique. Paris, SELAF. (Ethnosciences).

Bahuchet S. 1992 - Esquisse de l'ethnoichtyologie des Yasa du Cameroun. Anthropos 87 : 511-520.

Berkes F. 1993 - Traditional Ecological Knowledge in perspective. In : Inglis J.T. (Ed.) Traditional Ecological Knowledge: Concepts and Cases. Canadian Museum of Nature and International Development Research Centre, Ottawa : 1-9.

Bignoumba G.-S. 1999 - La politique maritime du Gabon à l'aube du troisième millénaire : l'indispensable ouverture sur la mer. Cahiers d'Outre-Mer 208 : 359-372.

Bouju S. 2000 - Activité de pêche et instrumentalisation des identités : pêcheurs migrants et pêcheurs nationaux dans la société guinéenne. In : Chauveau J.-P., Jul-Larsen E. \& Chaboud C. (Ed.) Les pêches piroguières en Afrique de l'Ouest. Paris, Karthala, CMI et IRD : 247-279.

Chauveau J.-P. \& Weber J. 1991 - L'apport des synthèses régionales. Perspectives historiques et institutionnelles sur la recherche interdisciplinaire dans le domaine des pêches artisanales. In : Durand J.-R., Lemoalle J. \& Weber J. (Ed.) La recherche face à la Pêche Artisanale. [Actes] Symp. Int. ORSTOM-IFREMER, Montpellier France, 3-7 juillet 1989. Paris, ORSTOM : 51-62.

Dia A. D. 2001 - Réseaux et groupes d'appartenance chez les pêcheurs migrants : le cas des Wolofs de N'Diago (Mauritanie). Bulletin Scientifique du Centre National de Recherches Océanographiques et des Pêches $28: 54-66$.

Doyon S. \& Sabinot C. (Ed.) 2015 - Anthropologie des espaces côtiers et de la conservation environnementale. Pêche, sel et flamants roses dans les Réserves de biosphère yucatèques au Mexique. Québec, Presses de l'Université de Laval.

Durand J.-R., Lemoalle J. \& Weber J. 1991 - La recherche face à la Pêche Artisanale. Symp. Int. ORSTOMIFREMER, Montpellier France, 3-7 juillet 1989. Paris, ORSTOM, 1076 p.

Friedberg C. 1997 - Diversité, ordre et unité du vivant dans les savoirs populaires. Natures Sciences Sociétés 5 (1) : 5-17.

Gelcich S., Gareth E.-J. \& Kaiser M. J. 2003 - Importance of attitudinal differences among artisanal fishers toward co-management and conservation of marine resources. Conservation Biology 19 (3) : 865-875.

Harvey D. 2002 - Agency and community: a critical realist paradigm. Journal for the theory of social behaviour 32 (2) : 163-194. 
Haudricourt A.-G. \& Dibie P. 1987 - Les pieds sur terre. Paris, A.-M. Métailié.

Hewlett B.S. \& Cavalli-Sforza L.L. 1986 - Cultural transmission among Aka Pygmies. American Anthropologist 88 (4) : 922-933.

Ingold T. 1993 - Temporality of landscape. World Archaeology 25 (2), « Conceptions of time and ancient society $»: 152-174$.

Jorion P. 1979 - Les deux concepts fondamentaux de la pêche artisanale : la « saison » et le « métier ». Ethnologie Française 9 (2) : 135-146.

Lave J. \& Wenger E. 1991 - Situated learning: legitimate peripheral participation. Cambridge, Cambridge University Press.

Marfaing L. 2005 - Du savoir faire sénégalais en matière de pêche sur les côtes mauritaniennes : une approche historique. Stichproben. Wiener Zeitschrift für kritische Afrikastudien. Vienna Journal of African Studies 8, « African Migrations. Historical Perspectives and contemporary Dynamics » : 69-98.

Patterson M. 2006 - Agency, kinship, and history in North Ambrym. Journal of the Royal Anthropological Institute 12 (1) : 211-217.

Reyes-García V., Martí N., MCDade T., Tanner S. \& VADEZ V. 2007 - Concepts and methods in studies measuring individual ethnobotanical knowledge. Journal of Ethnobiology 27 (2) : 182-203.

Reyes-García V., Paneque-Gálvez J., Luz A.C., Gueze M., Macía M.J., orta-Martínez M. \& Pino J. 2014 Cultural change and traditional ecological knowledge. An empirical analysis from the Tsimane' in the Bolivian Amazon. Human organization 73 (2) 162-173.

Rouch J. 1950 - Les Sorkawa Pêcheurs Itinérants du Moyen Niger. Journal of the International African Institute 20 (1) : 5-25.

Sabinot C. 2007 - Des hommes, des cultures, des savoirs et des savoir-faire en mouvement sur le littoral gabonais. Dynamique des savoirs et savoir-faire. In : Rouillard P. (Ed.) Mobilités, immobilismes. L'emprunt et son refus. Paris, De Boccard : 69-82.

Sabinot C. 2008 - Dynamique des savoirs et des savoir-faire dans un contexte pluriculturel. Étude comparative des activités littorales au Gabon. Thèse de Doctorat sous la direction de Serge Bahuchet. Paris, Muséum national d'Histoire naturelle. [En ligne :] http://tel.archives-ouvertes.fr/ tel-00326566/fr/

sabinot C. \& Bernard s. 2016 - An emblematic marine species at a crossroads in New Caledonia: Green Turtle. In : Fache E. \& Pauwels S. (Ed.) Resources, boundaries and governance: What future for fisheries in the Pacific? Pacific-credo Publications : 199-220.

sabinot c. \& Herrenschmidt J.B. 2019 - La dynamique des pratiques change-t-elle la manière de penser les relations kanak aux continuités terre-mer et à la nature ? Anthropologie et sociétés «Alternatives locales à la conservation environnementale » 43 (3).

Sabinot C. \& Lescureux N. 2019 - The Local ecological knowledge and the viability of the relations with the environment. In : Barrière O., Behnassi M., David G., Douzal V., Fargette M., Libourel T., Loireau M., Pascal L., Prost C., Ravena Cañete V., Seyler F. \& Morand S. (Ed.) Co-viability of Social and Ecological Systems : Reconnecting Mankind to the Biosphere in a Era of Global Change, Vol 1 The foundations of a new paradigm. Springer Nature Editions : 211-222.

Soengas B. 2010 - La subsistance des Pygmées Bakoya à l'épreuve de l'agriculture: dynamique des savoirs ethnobotaniques et des pratiques (Département de la Zadié, Ogooué-Ivindo, Gabon). Thèse de Doctorat, 
Sciences de l'Homme et Société. Paris, Museum national d'histoire naturelle. https://tel.archivesouvertes.fr/tel-00480270/

\section{NOTES}

1. Une ethnoécologie qui permet de poser un regard global sur les interactions dynamiques entre les humains et les non-humains (Bahuchet 1985 et 1992, Berkes 1993, Friedberg 1997, Haudricourt \& Dibie 1987).

2. (C) Sabinot, suite au travail prospectif le long des côtes gabonaises en 2004 et 2005 ; fonds de carte extrait de Demay 1998. Grand Atlas Larousse, Larousse-Bordas, Paris.

3. Quelques Punu résident dans la zone, soit à Mayumba même soit sur la route vers Tchibanga. Ils sont plus forestiers que leurs hôtes et le disent : «Je suis Bapunu. Moi,je ne connais pas l'eau. Moi, c'est les pièges. "

4. Quelques Pédah résident aux côtés des Phla. Tous sont appelés «Popo » par les autochtones.

5. L'identification des toponymes s'est réalisée sur deux années, en glanant régulièrement des informations auprès de la population, en réalisant un « tour virtuel » de la lagune Banio pendant une semaine de mai 2005 depuis la concession du vieux Nodet, homme né vers 1913 qui a patiemment sollicité ses souvenirs (identification de 162 toponymes de cette manière), puis en accomplissant une mission de trois jours en mars 2006 avec ce même homme et son fils (relevé toponymique - GPS, nom et histoire - d'un total de 301 sites).

6. Ce concept a été largement développé par Lave et Wenger (1991).

7. Cf. (Harvey 2002, Patterson 2006). Voir aussi (Gelcich et al. 2003) même si ces derniers n'utilisent pas le terme "agency». Dans les écrits de langue française, agency est parfois traduit par agencité ou agencéité.

8. Il convient de noter que toute compréhension fine des relations aux non-humains doit dépasser le caractère assurément réducteur de ce choix et s'est aussi appuyée sur de longs entretiens avec les interlocuteurs.

9. Pour en savoir plus sur la manière de mobiliser l'outil, se référer au chapitre 3 de la thèse de Sabinot (2008).

\section{RÉSUMÉS}

«Défier les approches disciplinaires", tout comme «l'apprentissage de l'océan", se fait seulement dans un contexte de confiance. Serge Bahuchet, qui a dirigé ma thèse de doctorat en ethnoécologie, a soutenu cette ambition scientifique qui consiste à aller au-delà de l'ethnographie et de l'anthropologie pour produire une compréhension approfondie des transformations sociétales. Le présent article décrit les choix méthodologiques réalisés pour décrire les changements qui ont marqué les habitants du littoral gabonais en matière de pêche et 
de rapport à la mer et propose une analyse de ce qui guide la dynamique des savoirs et savoirfaire des pêcheurs du Gabon.

"Challenge disciplinary approaches", as well as "ocean learning ", is only done in a context of trust. Serge Bahuchet, who supervised my PhD thesis in ethnoecology, supported scientific challenge which consists in going beyond ethnography and anthropology to produce a deep understanding of societal transformations. This paper describes the methodological choices made to describe the changes that have been observed on Gabonese coast in terms of fishing and interactions with the sea and to provide an analysis the dynamics of the knowledges and knowhow of Gabon's fishers.

\section{INDEX}

Keywords : Gabon, interdisciplinarity, fishers, cultural dynamics, knowledge transmission Mots-clés : Gabon, interdisciplinarité, pêcheurs, dynamiques culturelles, transmission des savoirs

\section{AUTEUR}

\section{CATHERINE SABINOT}

Chercheuse ethnoécologue et anthropologue - Centre IRD Anse Vata - BPA5 98848 Nouméa Cedex

- Nouvelle-Calédonie - UMR 228 ESPACE-DEV - Approche intégrée des milieux et des sociétés 